\section{Intensive serial biomarker profiling for the prediction of neutropenic fever in patients with hematologic malignancies undergoing chemotherapy: a pilot study}

\author{
Steven M. Chan, ${ }^{1}$ John Chadwick, ${ }^{2}$ \\ Daniel L. Young, ${ }^{3}$ Elizabeth Holmes, ${ }^{3}$ \\ Jason Gotlib \\ 1'Division of Hematology, Department of \\ Medicine, Stanford University School of \\ Medicine/Stanford Cancer Institute, CA, \\ USA; ${ }^{2}$ North Western Deanery, Three \\ Piccadilly Place, Manchester, UK; \\ ${ }^{3}$ Theranos Inc., Palo Alto, CA, USA
}

\section{Abstract}

Neutropenic fever (NF) is a life-threatening complication of myelosuppressive chemotherapy in patients with hematologic malignancies and triggers the administration of broadspectrum antimicrobials. The ability to accurately predict $\mathrm{NF}$ would permit initiation of antimicrobials earlier in the course of infection with the goal of decreasing morbid complications and progression to septic shock and death. Changes in the blood level of inflammatory biomarkers may precede the occurrence of NF. To identify potential biomarkers for the prediction of NF, we performed serial measurements of nine biomarkers [C-reactive protein (CRP), protein C, interleukin (IL)-6, IL-8, IL-10, IL-1 $\beta$, tumor necrosis factor- $\alpha$, monocyte chemotactic protein-1, and intercellular adhesion molecule-1] using a multiplex ELISA array platform every 6-8 hours in patients undergoing myelosuppressive chemotherapy for hematologic malignancies. We found that the blood levels of IL- 6 and CRP increased significantly 24 to 48 hours prior to the onset of fever. In addition, we showed that frequent biomarker monitoring is feasible using a bedside micro sample test device. The results of this pilot study suggest that serial monitoring of IL-6 and CRP levels using a bedside device may be useful in the prediction of NF. Prospective studies involving a larger cohort of patients to validate this observation are warranted. This trial is registered at ClinicalTrials.gov (NCT01144793).

\section{Introduction}

Patients undergoing induction chemotherapy for acute leukemias are at a particularly high risk of developing neutropenic fever (NF) due to the prolonged duration of severe neutropenia. In the majority of these cases, the rapid administration of broad spectrum antimicrobials decreases the risk of progression to septic shock and death. Despite advances in antimicrobial therapy and supportive care measures, infection-related complications frequently prolong the duration of hospitalization and continue to be the main causes of early mortality in patients undergoing chemotherapy for acute leukemias., Strategies to further decrease these complications are needed to improve patient outcomes.

The initiation of broad-spectrum antimicrobials for NF generally occurs at the time of fever. Although most patients stabilize after starting treatment, a subgroup of patients deteriorates rapidly into septic shock despite prompt initiation of antimicrobials. A strategy to improve outcomes is the use of antibiotic prophylaxis which has been shown to reduce infection-related mortality, especially with the use of fluoroquinolones. ${ }^{3}$ However, antibiotic resistance, fungal overgrowth, and antibioticrelated adverse effects (e.g. Clostridium difficile colitis and allergic responses) have limited the routine use of antibiotic prophylaxis. An alternative strategy is to initiate antimicrobial therapy just prior to the onset of fever. This strategy may reduce the incidence of infectionrelated complications by treating at an earlier time point when the pathogen burden is lower and the patient's condition is relatively more stable. To successfully implement this strategy, a practical method for the prediction of NF is required but no such method currently exists.

Pro-inflammatory cytokines such as interleukin (IL)-6, IL-1 family members, and tumor necrosis factor-alpha (TNF- $\alpha$ ) substantially increase in response to systemic infections. When they reach the central nervous system, they are able to increase the temperature set point and cause fever through induction of central mediators such as prostaglandins. ${ }^{4}$ Given that a threshold concentration of these cytokines is required before a fever response is initiated, we hypothesize that increases in their blood concentration should precede the onset of NF. Previous studies have focused mainly on the use of biomarkers measured at the time of NF for the prediction of adverse outcomes such as bacteremia or septic shock. ${ }^{5}$ In contrast, only a small number of studies have investigated the utility of biomarkers for the prediction of $\mathrm{NF}^{6-13} \mathrm{~A}$ few of these studies reported an increase in IL-6 and IL-8 levels before the onset of fever. ${ }^{6,11-13}$ A common limitation with all these studies is the low frequency of measurement, ranging from three times per week to at most once daily. This limitation may obscure the predictive potential of a biomarker if the change occurs temporally close to the onset of fever.
Correspondence: Jason Gotlib, Division of Hematology, Stanford University School of Medicine/Stanford Cancer Institute, 875 Blake Wilbur Drive, Room 2324, Stanford, CA 943055821, USA.

Tel.: +1.650.725.0744 - Fax: +1.650.724.5203.

E-mail: jason.gotlib@stanford.edu

Key words: neutropenic fever, interleukin-6, C-reactive protein, acute leukemia.

Acknowledgements: the authors would express gratitude to the Stanford Division of Hematology and Stanford University Hospital hematologyoncology nursing staff for their dedicated time and effort on this study. They also express gratitude to Dr. Stephen 0'Brien for his mentoring of Dr. John Chadwick

Contributions: SMC and JC are the first coauthors; SMC, JC and JG contributed to all aspects the study including study design, biomarker measurements, data analysis, and preparation of the manuscript; DLY and EH were involved in the measurement of biomarkers using the Theranos micro sample test.

Conflict of interests: the authors have no potential conflict of interests to disclose. This pilot study was a collaboration between Stanford University School of Medicine/Stanford Cancer Institute and Theranos, Inc, and no funding was received from Theranos for its implementation or conduct.

Received for publication: 26 April 2014.

Revision received: 5 June 2014.

Accepted for publication: 9 June 2014.

This work is licensed under a Creative Commons Attribution NonCommercial 3.0 License (CC BYNC 3.0).

(C) Copyright S.M. Chan et al., 2014

Licensee PAGEPress, Italy

Hematology Reports 2014; 6:5466

doi:10.4081/hr.2014.5466

We conducted a pilot study (ClinicalTrials. gov identifier NCT01144793) to assess the feasibility of frequent serial monitoring of blood biomarkers for the prediction of NF in hospitalized patients undergoing intensive chemotherapy for hematologic malignancies. We selected a panel of biomarkers that have previously been shown to be elevated in response to an infection and are thus most likely to rise prior to the onset of fever. ${ }^{14-23}$ We also included protein $\mathrm{C}$ which in contrast to the other biomarkers, has been shown to be decreased in the setting of sepsis and therefore its level may drop prior to fever onset. ${ }^{24}$ The study had two aims: i) to identify potential predictive biomarkers that are worthy of further study in a larger cohort to predict NF, and ii) to assess the feasibility of frequent monitoring of blood biomarkers for future implementation of a bedside device. 


\section{Materials and Methods}

\section{Patients}

Disease-specific eligibility criteria included adult patients ( $\geq 18$ years) with acute myeloid or lymphoid leukemia who were evaluated in the Stanford outpatient infusion treatment area (ITA) or inpatient hematology service. Patients undergoing induction or consolidation chemotherapy from April to June in 2008 were enrolled in the study. In our study, we defined neutropenia as an absolute neutrophil count (ANC) $<0.5 \times 10^{9} / \mathrm{L}$ and fever as a single body temperature greater than $38^{\circ} \mathrm{C}$. This definition was chosen because it would trigger the initiation of broad-spectrum antibiotics on our inpatient service. Enrolled patients were required to have an indwelling catheter in place before sampling could begin. Vitals signs were measured at least once every 8 hours and more frequently depending on the patients' clinical status. This study received approval from the Stanford Institutional Review Board (IRB) and the Stanford Cancer Institute Scientific Review Committee (SRC). A full written informed consent was obtained from all patients.

\section{Blood sampling and biomarker measurements}

Whole blood collection from the patient's central venous access line was initiated promptly following informed consent. One milliliter of blood was collected into an EDTA tube for each time point. The protocol specified collection of samples every 8 hours before the onset of fever and within 2 hours after fever developed. Sample collection changed to every 6 hours while patients were febrile, and reverted to every 8 hours after patients defervesced and the ANC rose above $1.0 \times 10^{9} / \mathrm{L}$. Blood was refrigerated at $4^{\circ} \mathrm{C}$ immediately upon collection and transported to an off-site central facility for further processing. The bulk of the sam- ple was centrifuged and the plasma fraction was aliquoted into $250 \mu \mathrm{L}$ barcoded vials. The plasma samples were then flash frozen in liquid nitrogen and stored at $-80^{\circ} \mathrm{C}$ for subsequent measurement of the biomarkers using Searchlight Protein Arrays (Pierce Biotechnology, Woburn, MA, USA). The following nine biomarkers were measured: $\mathrm{C}$-reactive protein (CRP), protein C, IL-6, IL-8, IL-10, IL-1 $\beta$, TNF- $\alpha$, monocyte chemotactic protein-1 (MCP-1), and intercellular adhesion molecule1 (ICAM-1). A small volume of whole blood was used for comparative analysis by a micro sample test (Theranos, Palo Alto, CA, USA). During this development and validation phase, analysis of samples using the micro sample test was performed at an off-site facility.

The micro sample processing and analytical system developed by Theranos consists of single-use consumables that require only a small volume of blood sample ( $25 \mu \mathrm{L})$ for analysis and instrumentation and software enabling serial multiplexed enzyme-linked immunosorbent assays of a wide spectrum of biomarkers. The sample preparation and assays are fully automated and analysis takes less than 1 hour to complete. In this study, CRP and protein C levels were measured using this system.

\section{Statistical analysis}

The level of significance in the difference between fold-changes for each biomarker was determined using the Wilcoxon signed rank test. This test compares the difference between matched pairs (distal vs proximal value of an individual patient) and does not assume the fold-change values to be normally distributed. A P-value less than 0.05 was considered statistically significant. The Pearson's correlation coefficient was used to determine strength of correlation between CRP levels measured using the Searchlight assay and the Theranos micro sample test. The GraphPad Prism software (version 6, GraphPad Software, La Jolla, CA, USA) was used for all calculations.

\section{Results and Discussion}

A total of seventeen patients were enrolled in this pilot study. The median age of the patients enrolled was 49 years (range: 22-70 years). Twelve patients (70\%) were male. The underlying diagnoses were acute myeloid leukemia $(\mathrm{n}=12)$, acute B- or T-lymphoblastic leukemia $(n=3)$, and chronic myeloid leukaemia in either lymphoid or myeloid blast crisis $(n=2)$. Two of the patients were monitored in the outpatient ITA for either all or part of the pre-fever portion of sampling. A total of 1302 samples were drawn over the study period representing $96.5 \%$ of the planned draws.

In order to determine the relative change in the level of a biomarker prior to the onset of fever, a baseline measurement from which all comparisons are made is required. The sample collected 5 to 7 days prior to the onset of first fever was used as the baseline in our study. The baseline measurement was not available in 5 patients and they were excluded for further analysis. One patient did not have a sample collected within 8 hours prior to onset of first fever and was also excluded. Logistical issues with blood sampling included the need for venous catheter line removal due to suspected line infections $(n=2)$ and patient refusal of blood draws $(n=1)$. For patients who were febrile at the time of enrollment, they were included if the fever resolved within 48 hours of enrollment and was followed by at least a 10-day afebrile interval before onset of the next fever. For this subgroup of patients, the second fever was used as the reference point for analysis. Four of the 17 patients did not meet this requirement and were excluded. An additional patient was excluded because he was not neutropenic at the time of fever. As a result, six of the seventeen patients (35\%) were considered fully evaluable.

of the six patients included for further analysis, five of them had acute myeloid leukemia and the remaining patient had B-cell acute lymphoblastic leukemia (Table 1). All the

Table 1. Characteristics of the patients included for analysis.

\begin{tabular}{lccccccc} 
ID & Age & Sex & Primary diagnosis & $\begin{array}{c}\text { Chemotherapy } \\
\text { regimen }\end{array}$ & $\begin{array}{c}\text { Chemotherapy } \\
\text { started on day }\end{array}$ & Day of fever & Source/site of infection \\
4 & 59 & M & B-ALL & CALGB 9511 & 0 & 6.8 & Bacteremia, K. pneumoniae \\
9 & 39 & F & AML & High dose cytarabine consolidation & -4 & 8.7 & Right upper lobe pneumonia \\
\hline 10 & 70 & M & Secondary AML & $3+7$ & 2 & 10.6 & Unknown \\
11 & 57 & M & AML & $3+7$ & 3 & 14.1 & Unknown \\
\hline 13 & 38 & F & AML & $3+4$ & 2 & 11.8 & Bacteremia, E. coli \\
15 & 52 & M & Secondary AML & $3+4$ & 8.8 & Unknown \\
\hline
\end{tabular}

AML, acute myeloid leukemia; B-ALL, B-cell acute lymphoblastic leukemia; CALGB, Cancer and Leukemia Group B.

For each patient, day 0 corresponds to the day of enrollment in this study. $3+7$ refers to a standard AML induction regimen consisting of 3 days of anthracycline and 7 days of continuous infusion cytarabine (100 $\mathrm{mg} / \mathrm{m} 2 /$ day $) .3+4$ is a similar regimen except cytarabine is given at high doses $(3 \mathrm{gm} / \mathrm{m} 2)$ twice daily for 4 days. 
patients received induction chemotherapy regimens except for patient \#9 who received consolidation therapy for AML. The median duration between the start of chemotherapy and subsequent fever was 8.7 days (range: 6.8-12.7 days). The median interval between blood draws in the pre-febrile period was 9 hours (range: 2-45 hours) and dropped to 7 hours (range: 2-24 hours) in the 72-hour period following the onset of fever. A source or site of infection was identified in three of the six patients (Table 1). Notably, patient \#4 developed septic shock requiring care in the intensive care unit as a result of a Klebsiella pneumoniae infection.

To identify candidate biomarkers with the potential to predict NF, we determined the foldchanges at two time points for each biomarker and patient. The first one, henceforth referred to as proximal, was the ratio of the measurement drawn just prior to the onset of fever to the baseline measurement collected 5 to 7 days prior to fever onset. The second one, henceforth referred to as distal, was the ratio of the measurement taken between 2.5 to 3.5 days prior to onset of fever to the day 5-7 baseline measurement. Since the concentration of a predictive biomarker should progressively increase over time prior to the onset of fever, the proximal fold-change should correspondingly be higher than the distal fold-change. Non-predictive biomarkers should not demon- strate a difference between the two values. Of the nine biomarkers measured, only IL- 6 and CRP had a statistically significant $(\mathrm{P}<0.05)$ difference between the proximal and distal fold-changes (Figure 1). The mean proximal fold-changes for IL-6 and CRP were 24.7 (range: 6.5-91) and 38.2 (range: 0.179-135) and mean distal fold-changes were 1.6 (range: 1.03-1.99) and 0.78 (range: $0.19-2.06$ ), respec- tively. All patients had a higher proximal than distal fold-change for IL- 6 . In contrast, 4 of the 6 patients showed the same trend for CRP.

We next determined the time at which IL-6 and CRP predicted the onset of fever. For each biomarker, the mean of the distal fold-changes plus two standard deviations was used as the cut-off value above which fever was predicted to occur. This method of determining the cut-

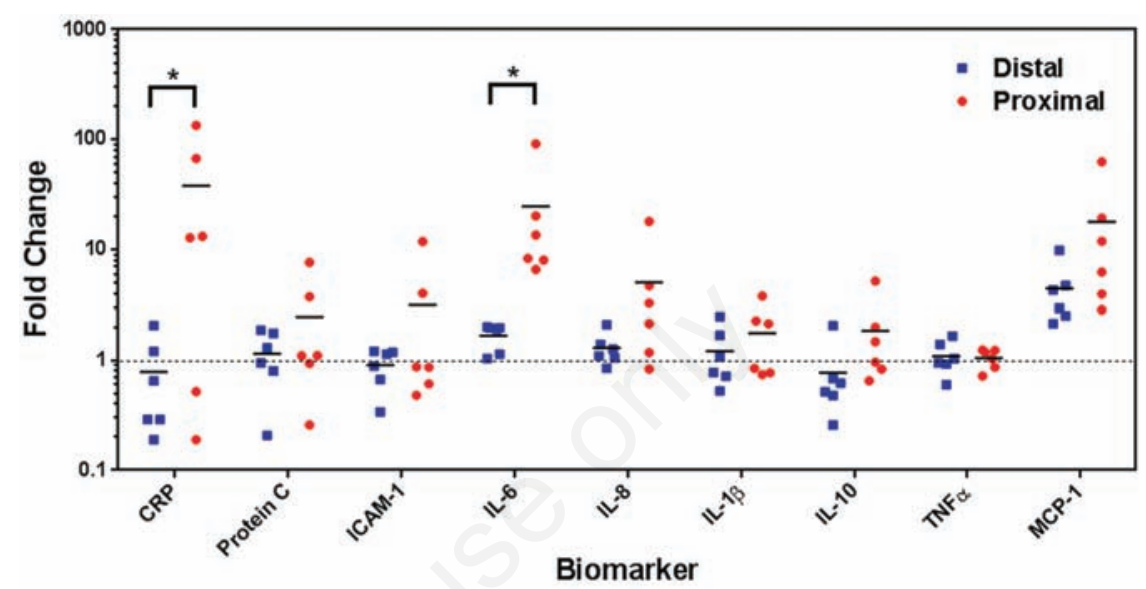

Figure 1. Scatter plots of the proximal and distal fold-changes for each biomarker. Each point represents data from an individual patient. The mean value (horizontal line) is shown. ${ }^{*} \mathrm{P}<0.05$.

Patient \#10
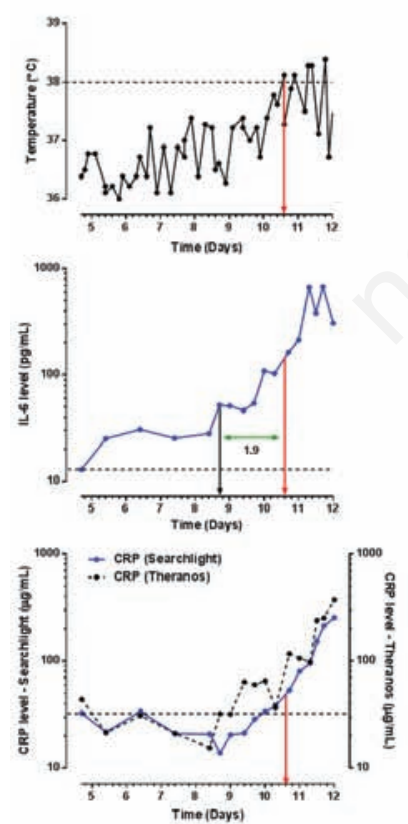

Patient \#11
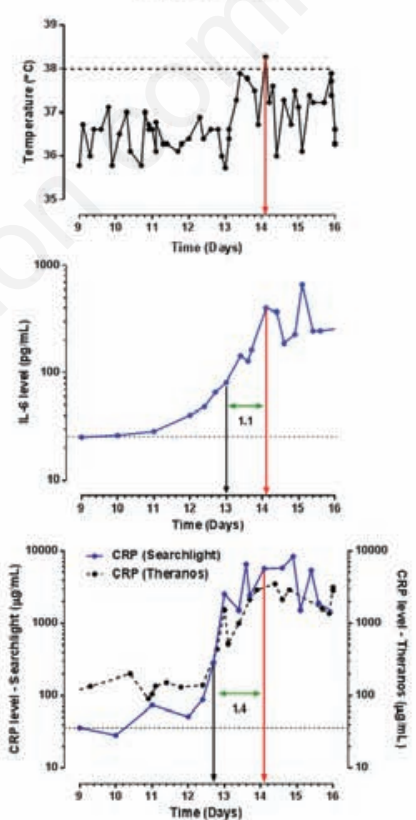

Patient \#13
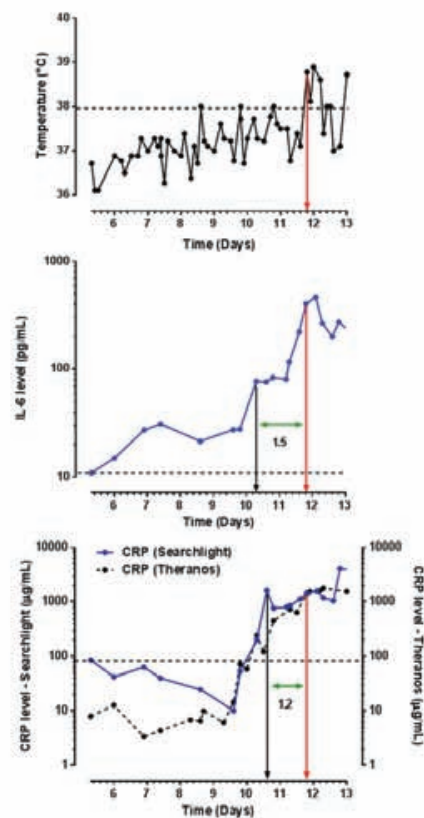

Patient \#15
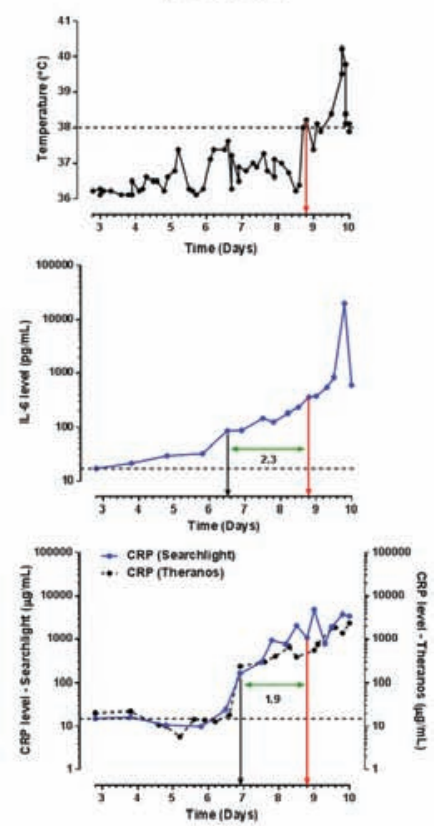

Figure 2. Time course of body temperature, interleukin (IL)-6 and C-reactive protein (CRP) levels. Data are shown starting from the time of baseline measurement (5-7 days prior to fever onset) to the time of fever. Day 0 corresponds to the day of study enrollment. Red arrow indicates the time of fever. Black arrow indicates the time when the fold-change cut-off is crossed. The number under the green line refers to the time difference in days between these two values. The dotted horizontal lines in the IL-6 and CRP graphs indicate the baseline Searchlight measurements prior to onset of fever. Refer to Results and Discussion for details. 
off assumes that the distal fold-changes reflected mainly physiologic variations in the concentration of the biomarker and were not a direct response to the inciting infection that eventually caused the fever. Using this approach, IL-6 levels crossed the cut-off value for all patients at a median of 1.3 (range: 0.4 2.3) days prior to the onset of fever. For CRP, the cut-off was crossed in 4 of the 6 patients at a median of 1.7 (range: 1.2-2.3) days before fever onset. A closer inspection of the fever curves of 4 of the study patients in conjunction with their IL-6 and CRP levels over time clearly demonstrates an upward trend of these biomarkers prior to the onset of fever (Figure 2). Taken together, our analysis provides evidence that IL-6 and CRP may be useful in predicting the onset of NF with a lead time of 1 to 2 days.

To demonstrate the feasibility of serial biomarker monitoring with the micro sample test system, we measured the level of CRP and protein $\mathrm{C}$ in parallel using the same patient samples. Because CRP proved to be a potentially useful predictive biomarker, we focused our analysis on this biomarker. CRP levels of the six fully evaluable patients measured using the micro sample test correlated highly with values obtained using the commercially available Searchlight Protein Arrays (Figure 3). The Pearson's correlation coefficient ( $r$ ) between the Theranos and Searchlight measurements was 0.85 . Measurements using the micro sample test also demonstrated a rise in CRP levels prior to the onset of fever (Figure 2). A larger study is required to determine if the micro sample test system can be used interchangeably with standard laboratory assays which would support its use to predict NF in the hospital setting.

Although several cytokines including IL-6, IL-1 family members, and TNF- $\alpha$ have been implicated in mediating the fever response, ${ }^{4}$ IL-6 was the only pro-inflammatory cytokine in our array screen that demonstrated promise in the prediction of NF. This finding suggests that IL-6 is a predominant cytokine mediating the febrile response in neutropenic patients. CRP, which is an acute phase reactant induced by IL- $6,{ }^{25}$ was correspondingly also predictive of $\mathrm{NF}$ in our study population. These results are in agreement with prior studies that examined changes in IL-6 and CRP levels. Engel et al. reported a significant increase in IL-6 levels 24 to 48 hours prior to onset of fever. ${ }^{6}$ They reported a shorter lead time for CRP of less than 24 hours. Two other studies reported a similar upward trend 24 to 48 hours before onset of fever with IL- $6{ }^{11,13}$ As mentioned previously, the frequency of measurement in these prior studies was only three times a week which may be insufficient for the prediction of NF on an individual basis. An advantage of our study is the relatively high frequency of measurements which increases the temporal

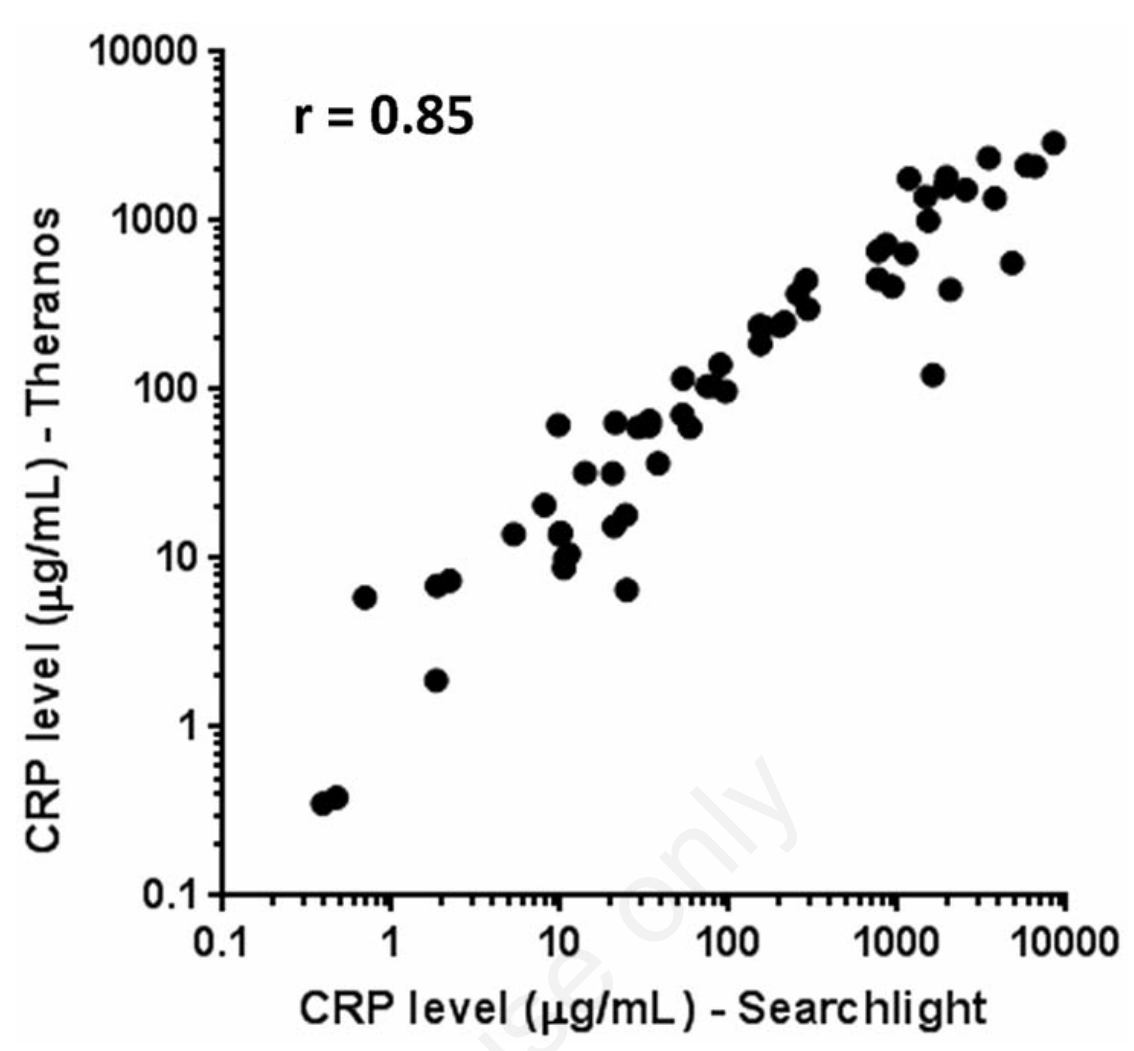

Figure 3. Plot of C-reactive protein levels measured using the Searchlight assay versus the Theranos micro sample test. Each data point represents an individual blood sample collected from one of the six fully evaluable patients in this study. The Pearson's correlation coefficient (r) is shown.

resolution of biomarker trends and demonstrates the predictive power of IL- 6 and CRP in individual patients. However, there were some challenges to the collection of blood samples at this high frequency including missed draws from nursing staff, the need for central line removal for suspected line infection, and infrequent patient refusal of blood draws. Improved communication with the nursing staff and patients as well as a more streamlined sample collection protocol should help overcome the challenges identified in this pilot study.

The ability to reliably predict the onset of NF may permit early implementation of broadspectrum antibiotics in order to reduce the likelihood of infection-related morbidity and mortality. Although the number of fully evaluable patients in this pilot, hypothesis-forming study was small, our results point to further exploration of the biomarkers IL-6 and CRP for prediction of NF. Future prospective studies with a larger cohort of patients using the micro sample test system for real-time measurements of these and additional biomarkers are needed to validate our results and determine the sensitivity and specificity of this approach.

\section{References}

1. Creutzig U, Zimmermann M, Reinhardt D, et al. Early deaths and treatment-related mortality in children undergoing therapy for acute myeloid leukemia: analysis of the multicenter clinical trials AML-BFM 93 and AML-BFM 98. J Clin Oncol 2004;22:4384-93.

2. Slats AM, Egeler RM, van der Does-van den Berg A, et al. Causes of death-other than progressive leukemia-in childhood acute lymphoblastic (ALL) and myeloid leukemia (AML): the Dutch Childhood Oncology Group experience. Leukemia 2005;19:537-44.

3. Gafter-Gvili A, Fraser A, Paul M, et al. Antibiotic prophylaxis for bacterial infections in afebrile neutropenic patients following chemotherapy. Cochrane Database Syst Rev 2012;1:CD004386.

4. Conti B, Tabarean I, Andrei C, Bartfai T. Cytokines and fever. Front Biosci 2004;9: 1433-49.

5. Phillips RS, Wade R, Lehrnbecher T, et al. Systematic review and meta-analysis of 
the value of initial biomarkers in predicting adverse outcome in febrile neutropenic episodes in children and young people with cancer. BMC Med 2012;10:6.

6. Engel A, Mack E, Kern P, Kern WV. An analysis of interleukin-8, interleukin-6 and C-reactive protein serum concentrations to predict fever, gram-negative bacteremia and complicated infection in neutropenic cancer patients. Infection 1998;26:213-21.

7. Goetz M, Behre G, Heussel G, et al. Soluble tumor necrosis factor receptor type II in the early diagnosis of fever in neutropenia. Ann Hematol 2002;81:382-5.

8. Engel A, Steinbach G, Kern P, Kern WV. Diagnostic value of procalcitonin serum levels in neutropenic patients with fever: comparison with interleukin-8. Scand J Infect Dis 1999;31:185-9.

9. Buyukberber N, Buyukberber S, Sevinc A, Camci C. Cytokine concentrations are not predictive of bacteremia in febrile neutropenic patients. Med Oncol 2009;26:55-61.

10. Ribeiro AF, Nobre V, Neuenschwander LC, et al. Use of inflammatory molecules to predict the occurrence of fever in oncohematological patients with neutropenia. Braz J Med Biol Res 2013;46:200-6.

11. Steinmetz HT, Herbertz A, Bertram M, Diehl V. Increase in interleukin-6 serum level preceding fever in granulocytopenia and correlation with death from sepsis. J Infect Dis 1995;171:225-8.

12. Lindemann A, Tamm I, Tanodi K, Mertelsmann R. Interleukin-8 serum levels for early detection of infectious episodes in neutropenic patients. J Infect Dis 1995; $172: 610$. P

13. Schonbohn H, Schuler M, Kolbe K, et al. Plasma levels of IL-1, TNF alpha, IL-6, IL-8, G-CSF, and IL1-RA during febrile neutropenia: results of a prospective study in patients undergoing chemotherapy for acute myelogenous leukemia. Ann Hematol 1995;71:161-8.

14. Netea MG, Kullberg BJ, Van der Meer JW. Circulating cytokines as mediators of fever. Clin Infect Dis 2000;31:S178-84.

15. Kruttgen A, Rose-John S. Interleukin-6 in sepsis and capillary leakage syndrome. J Interferon Cytokine Res 2012;32:60-5.

16. Kunkel SL, Lukacs NW, Strieter RM. The role of interleukin- 8 in the infectious process. Ann N Y Acad Sci 1994;730:134-43.

17. Du Clos TW, Mold C. The role of C-reactive protein in the resolution of bacterial infection. Curr Opin Infecti Dis 2001;14:289-93.

18. Scumpia P0, Moldawer LL. Biology of interleukin-10 and its regulatory roles in sepsis syndromes. Crit Care Med 2005;33: S468-71.
19. Oberholzer A, Oberholzer C, Moldawer LL. Interleukin-10: a complex role in the pathogenesis of sepsis syndromes and its potential as an anti-inflammatory drug. Crit Care Med 2002;30:S58-63.

20. Pruitt JH, Copeland EM 3rd, Moldawer LL. Interleukin-1 and interleukin-1 antagonism in sepsis, systemic inflammatory response syndrome, and septic shock. Shock 1995;3:235-51.

21. Zentella A, Manogue K, Cerami A. The role of cachectin/TNF and other cytokines in sepsis. Prog Clin Biol Res 1991;367:9-24.

22. Gomes RN, Teixeira-Cunha MG, Figueiredo RT, et al. Bacterial clearance in septic mice is modulated by MCP-1/CCL2 and nitric oxide. Shock 2013;39:63-9.

23. Figueras-Aloy J, Gomez-Lopez L, RodriguezMiguelez JM, et al. Serum soluble ICAM-1, VCAM-1, L-selectin, and P-selectin levels as markers of infection and their relation to clinical severity in neonatal sepsis. Am J Perinatol 2007;24:331-8.

24. Fisher CJ Jr., Yan SB. Protein C levels as a prognostic indicator of outcome in sepsis and related diseases. Crit Care Med 2000;28:S49-56.

25. Eklund CM. Proinflammatory cytokines in CRP baseline regulation. Adv Clin Chem 2009;48:111-36. 